\title{
Tilting in module categories
}

\author{
Robert Wisbauer \\ Mathematical Institute of the University \\ 40225 Düsseldorf, Germany
}

\begin{abstract}
Let $M$ be a module over an associative ring $R$ and $\sigma[M]$ the category of $M$-subgenerated modules. Generalizing the notion of a projective generator in $\sigma[M]$, a module $P \in \sigma[M]$ is called tilting in $\sigma[M]$ if (i) $P$ is projective in the category of $P$-generated modules, (ii) every $P$-generated module is $P$ presented, and (iii) $\sigma[P]=\sigma[M]$. We call $P$ self-tilting if it is tilting in $\sigma[P]$. Examples of (not self-small) tilting modules are $\mathscr{Q} / \mathbb{Z}$ in the category of torsion $\mathbb{Z}$-modules, $Q \oplus Q / \mathbb{Z}$ in the category $\mathbb{Z}$-Mod, certain divisible modules over integral domains, and also cohereditary coalgebras $C$ over a QF-ring in the category of comodules over $C$. Self-small tilting modules $P$ in $\sigma[M]$ are finitely presented in $\sigma[M]$. For $M=P$, they are just the $*$-modules introduced by C. Menini and A. Orsatti, and for $M=R$, they are the usual tilting modules considered in representation theory.

Notice that our techniques and most of our results also apply to locally finitely generated Grothendieck categories.
\end{abstract}

\section{Introduction}

Tilting modules $P$ were first defined in representation theory of finite-dimensional algebras $R$ by the conditions

(1) $\operatorname{Ext}_{R}^{1}\left(P, P^{(\Lambda)}\right)=0$, for any set $\Lambda$,

(2) $\operatorname{Ext}_{R}^{2}(P, N)=0$, for all $N \in R$-Mod,

(3) there exists an exact sequence $0 \rightarrow R \rightarrow P_{1} \rightarrow P_{2} \rightarrow 0$, with $P_{1}, P_{2} \in$ add $(P)$,

(4) $P$ is finitely generated.

The interest in these modules stems from the fact that they allow generalizations of Morita equivalences. 
Although it is clear that any progenerator $P$ is tilting, it is not so obvious which one of the conditions generalizes projectivity and which one the generator property. Moreover one may ask if a generalization of the simply defined properties of a progenerator really needs techniques from homological algebra to be formulated.

It will turn out that (1), (2), (3) are in fact equivalent to the properties

(i) $P$ is projective in the category of $P$-generated modules,

(ii) every $P$-generated module is $P$-presented,

(iii) $P$ is a subgenerator in $R$-Mod.

Here the first two properties are only related to the category $\operatorname{Gen}(P)$ of $P$ generated modules, whereas (iii) gives a relation of $P$ to $R$-Mod. More generally, for an $R$-module $M$, we call $P$ a tilting module in the category $\sigma[M]$ of $M$-subgenerated modules, if it satisfies (i), (ii) and if $P$ is a subgenerator of $\sigma[M]$. Taking $M=R$ we are back in $R$-Mod. Since $P$ is always a subgenerator in $\sigma[P], P$ is tilting in $\sigma[P]$ if (i) and (ii) are satisfied. Such modules are called self-tilting. To widen the range of applications we do not demand a tilting module to be finitely generated (compare Facchini [10], Colpi-Trlifaj [6]).

We will see that $Q / \mathbb{Z}$ is a tilting module in the category of torsion $\mathbb{Z}$-modules (= $\sigma[Q / \mathbb{Z}]), \mathbb{Q} \oplus \mathscr{Q} / \mathbb{Z}$ is tilting in $\mathbb{Z}$-Mod, and over an integral domain $R$, the divisible module $\partial$ introduced in Fuchs [12] is tilting in $R$-Mod. Moreover any cohereditary coalgebra $C$ over a QF-ring is tilting in the category of comodules over $C$.

It turns out that self-small self-tilting modules $P$ are precisely the $*$-modules introduced by Menini und Orsatti [15] (named in Colpi [2]). This observation originally resulted from discussions on the subject with $\mathrm{R}$. Colpi and W. Folz. It was the main motivation for this paper, which is an analysis and extension of the work of C. Menini, A. Orsatti, R. Colpi, J. Trlifaj and others on the subject. Some of the results already appear in one or the other form in their papers. Nevertheless we provide complete proofs (modulo standard knowledge in module theory) which simplify existing ones.

After this paper was essentially finished we received the preprint [4] of R. Colpi which provides alternative proofs for some of our results (see 5.4).

\section{Preliminaries}

Let $R$ denote an associative ring with unit and $R$-Mod the category of unital left $R$-modules. Homomorphisms of modules will usually be written on the opposite side of the scalars. For unexplained notation the reader is referred to [19].

Let $M \in R$-Mod. An $R$-module $N$ is $M$-generated if there exists an exact sequence

$$
0 \rightarrow K \rightarrow M^{(\Lambda)} \rightarrow N \rightarrow 0, \quad \Lambda \text { some set }
$$


and $N$ is (semi-finitely) $M$-presented if there exists such a sequence (with $\Lambda$ finite) where $K$ is $M$-generated.

$\operatorname{Gen}(M), \operatorname{Pres}(M), \operatorname{Pres}_{f}(M)$ and $\sigma[M]$ will denote the full subcategories of $R$-Mod whose objects are $M$-generated, $M$-presented, semi-finitely $M$-presented or submodules of $M$-generated modules, respectively. Add $(M)(\operatorname{resp}$. add $(M))$ stands for the class of modules which are direct summands of (finite) direct sums of copies of $M$. We have

$$
\operatorname{add}(M) \subset \operatorname{Add}(M) \subset \operatorname{Pres}(M) \subset \operatorname{Gen}(M) \subset \sigma[M] .
$$

Obviously $M$ is a generator in $\sigma[M]$ if and only if $\operatorname{Pres}(M)=\operatorname{Gen}(M)=\sigma[M]$. A module $N \in \sigma[M]$ is a subgenerator in $\sigma[M]$ if $\sigma[N]=\sigma[M]$. A progenerator in $\sigma[M]$ is a finitely generated projective generator in $\sigma[M]$.

$\sigma[M]$ is closed under direct sums, factor modules and submodules in $R$-Mod (hence it is a Grothendieck category). Notice that for any family $\left\{N_{\lambda}\right\}_{\Lambda}$ of modules in $\sigma[M]$ there exists a product in $\sigma[M]$ but it differs from the product in $R$-Mod (cartesian product). This product we denote by (see $[19,15.1]$ )

$$
\prod_{\Lambda}^{M} N_{\lambda}=\operatorname{Tr}\left(\sigma[M], \prod_{\Lambda} N_{\lambda}\right), \text { and }\left.N\right|_{M} ^{\Lambda}=\operatorname{Tr}\left(\sigma[M], N^{\Lambda}\right)
$$

where the right side is the short form for the case $N_{\lambda}=N$, for all $\lambda \in \Lambda$. Notice that for any $P \in \sigma[M]$, we have

$\operatorname{Hom}_{R}\left(P, \prod_{\Lambda}^{M} N_{\lambda}\right) \simeq \prod_{\Lambda} \operatorname{Hom}_{R}\left(P, N_{\lambda}\right)$, and $\operatorname{Hom}_{R}\left(P,\left.N\right|_{M} ^{\Lambda}\right) \simeq \operatorname{Hom}_{R}(P, N)^{\Lambda}$.

Generating properties of $P$ will be of great interest in our investigations. As a first instance we show:

\section{$2.1 P$ generates $\left.P\right|_{M} ^{\Lambda}$.}

Assume there is a progenerator $G$ in $\sigma[M]$. Let $P \in \sigma[M]$ and $S=\operatorname{End}_{R}(P)$. Then:

(1) If $P$ generates $\left.P\right|_{M} ^{\Lambda}$, for any $\Lambda$, then $\operatorname{Hom}_{R}(G, P)_{S}$ is finitely generated.

(2) If $P$ is a generator in $\sigma[M]$, then $\operatorname{Hom}_{R}(G, P)_{S}$ is finitely generated.

(3) If $P$ is self-injective and $G \in \sigma[P]$, then $\operatorname{Hom}_{R}(G, P)_{S}$ is finitely generated.

Proof. (1) Consider the canonical map

$$
\varphi: \operatorname{Hom}_{R}(G, P) \otimes_{S} S^{\Lambda} \rightarrow \operatorname{Hom}_{R}(G, P)^{\Lambda}, \quad h \otimes\left(s_{\lambda}\right)_{\Lambda} \mapsto\left(h s_{\lambda}\right)_{\Lambda} .
$$

There is an epimorphism $\alpha:\left.P^{(\Omega)} \rightarrow P\right|_{M} ^{\Lambda}$, and for $f \in \operatorname{Hom}_{R}\left(G,\left.P\right|_{M} ^{\Lambda}\right) \simeq \operatorname{Hom}_{R}(P, N)^{\Lambda}$, the diagram

$$
\begin{array}{rc} 
& G \\
& \\
& \downarrow f \\
\left.P^{(\Omega)} \stackrel{\alpha}{\rightarrow} \quad P\right|_{M} ^{\Lambda},
\end{array}
$$


can be extended commutatively by some $h: G \rightarrow P^{(\Omega)}$. Denoting by $\varepsilon_{\lambda}$ and $\pi_{\lambda}$ the canonical injections and projections, we have

$$
f=\sum_{i=1}^{k}\left(h \pi_{\lambda_{i}}\right)\left(\varepsilon_{\lambda_{i}} \alpha\right) \text {, where } h \pi_{\lambda_{i}} \in \operatorname{Hom}_{R}(G, P), \varepsilon_{\lambda_{i}} \alpha \in S^{\Lambda} .
$$

This shows that $\varphi$ is surjective and hence $\operatorname{Hom}_{R}(G, P)_{S}$ is finitely generated (e.g., $[19,12.9])$.

(2) is a special case of (1).

(3) If $P$ is self-injective, then $\left.P\right|_{P} ^{\Lambda}$ is injective in $\sigma[P]$ and hence $P$-generated. Now (1) applies.

Putting $M=R$ the above observations yield the well known fact that generators as well as injective subgenerators in $R$-Mod are finitely generated as modules over their endomorphism ring.

Although there may be no projectives in $\sigma[M]$ there are enough injectives and the Ext-functor can be defined from injective resolutions.

2.2 Ext-functor in $\sigma[M]$.

By $\operatorname{Ext}_{M}^{1}$ and $\operatorname{Ext}_{M}^{2}$ we denote the first and second Ext-functor in $\sigma[M]$. So for $P \in \sigma[M]$ and any exact sequence $0 \rightarrow K \rightarrow L \rightarrow N \rightarrow 0$ in $\sigma[M]$, we have the long exact sequence

$$
\begin{aligned}
0 \rightarrow & \operatorname{Hom}_{R}(P, K) \rightarrow \operatorname{Hom}_{R}(P, L) \rightarrow \operatorname{Hom}_{R}(P, N) \rightarrow \\
& \operatorname{Ext}_{M}^{1}(P, K) \rightarrow \operatorname{Ext}_{M}^{1}(P, L) \rightarrow \operatorname{Ext}_{M}^{1}(P, N) \rightarrow \operatorname{Ext}_{M}^{2}(P, K) \rightarrow \cdots .
\end{aligned}
$$

For $P \in \sigma[M]$ we denote the kernel of $\operatorname{Ext}_{M}^{1}(P,-)$ by

$$
P^{\perp_{M}}:=\left\{N \in \sigma[M] \mid \operatorname{Ext}_{M}^{1}(P, N)=0\right\} .
$$

Putting $M=R$ the functors $\operatorname{Ext}_{R}^{1}$ and $\operatorname{Ext}_{R}^{2}$ yield the familiar functors for $R$-Mod and $P^{\perp_{R}}$ denotes the kernel of $\operatorname{Ext}_{R}^{1}(P,-)$.

For subgenerators we have various characterizations ([19, Section 15 and 16.3]):

\subsection{Subgenerators.}

(1) For $N \in \sigma[M]$ the following are equivalent:

(a) $N$ is a subgenerator in $\sigma[M]$;

(b) $N$ generates all injective modules in $\sigma[M]$;

(c) $N$ generates the $M$-injective hull $\widehat{M}$ of $M$.

If $\sigma[M]$ has a progenerator $G$, then (a)-(c) are equivalent to: 
(d) there exists a monomorphism $G \rightarrow N^{k}$, for some $k \in \mathbb{N}$.

(2) For an $R$-module $M$ the following are equivalent:

(a) $M$ is a subgenerator in $R$-Mod (i.e., $\sigma[M]=R$-Mod);

(b) $M$ generates all injective modules in $R$-Mod;

(c) $M$ generates the injective hull $E(R)$ of $R$;

(d) there is a monomorphism $R \rightarrow M^{k}$, for some $k \in \mathbb{N}$.

(3) A faithful $R$-module ${ }_{R} M$ is a subgenerator in $R$-Mod provided

(i) ${ }_{R} M$ is finitely generated over $\operatorname{End}_{R}(M)$, or

(ii) ${ }_{R} R$ is finitely cogenerated, or

(iii) ${ }_{R} M$ is finitely generated and $R$ is commutative, or

(iv) $\sigma[M]$ is closed under products in $R$-Mod.

A finitely generated module $N \in \sigma[M]$ is finitely presented in $\sigma[M]$ if for any exact sequence

$$
0 \rightarrow K \rightarrow L \rightarrow N \rightarrow 0
$$

in $\sigma[M], L$ finitely generated implies that $K$ is finitely generated.

$N$ is called weakly $M$-injective if $\operatorname{Hom}_{R}(-, N)$ turns monomorphisms $K \rightarrow M^{(\mathbb{N})}$ to epimorphisms, provided $K$ is finitely generated.

\subsection{Finitely presented modules in $\sigma[M]$.}

(1) For a finitely generated $P \in \sigma[M]$, the following are equivalent:

(a) $P$ is finitely presented in $\sigma[M]$;

(b) $\operatorname{Hom}_{R}(P,-)$ commutes with direct limits in $\sigma[M]$;

(c) $\operatorname{Hom}_{R}(P,-)$ commutes with direct limits of $M$-generated modules;

(d) $\operatorname{Hom}_{R}(P,-)$ commutes with direct limits of weakly $M$-injective modules.

(2) If $P$ is finitely presented in $\sigma[P]$ then $P^{\perp_{M}}$ is closed under direct sums.

(3) For any module $P$ the following are equivalent:

(a) $P$ is finitely presented in $\sigma[P]$;

(b) $\operatorname{Hom}_{R}(P,-)$ commutes with direct limits of P-generated modules.

Proof. $(1)(a) \Leftrightarrow(b) \Leftrightarrow(d)$ are shown in $[19,25.2])$.

$(c) \Leftrightarrow(d)$ is clear by the fact that weakly $M$-injective modules are $M$-generated (by $[19,16.11]$ ). 
(2) Let $\left\{K_{\lambda}\right\}_{\Lambda}$ be a family of modules in $P^{\perp_{M}}$. Taking any extension of $P$ by $\oplus_{\Lambda} K_{\lambda}$ as lower sequence we construct an exact commutative diagram

$$
\begin{aligned}
& \begin{array}{lllllll}
0 & \rightarrow & K & \stackrel{f}{\rightarrow} & L & \rightarrow & P
\end{array} \quad \rightarrow \quad 0 \\
& 0 \rightarrow \oplus_{\Lambda} K_{\lambda} \rightarrow Q \rightarrow P \rightarrow 0 \text {, }
\end{aligned}
$$

where $L$ is some finitely generated submodule of $Q$ and $K$ is finitely generated. So $\operatorname{Im} g$ is contained in a finite partial sum of $\bigoplus_{\Lambda} K_{\lambda}$ and there exists some morphism $h: L \rightarrow \oplus_{\Lambda} K_{\lambda}$ with $f h=g$. Now it follows by the Homotopy Lemma that the lower sequence splits and so $\bigoplus_{\Lambda} K_{\lambda} \in P^{\perp_{M}}$.

(3) By (1), it remains to show that (b) implies that $P$ is finitely generated.

Let $\left\{K_{i}\right\}_{I}$ denote the direct family of finitely generated submodules of $P$. For $i \in I$ we have the exact exact sequence

$$
0 \rightarrow K_{i} \rightarrow P \rightarrow P / K_{i} \rightarrow 0
$$

and $\left\{P / K_{i}\right\}_{I}$ form a direct system of $P$-generated modules. Since $\operatorname{Hom}_{R}(P,-)$ commutes with its direct limit we obtain an exact sequence

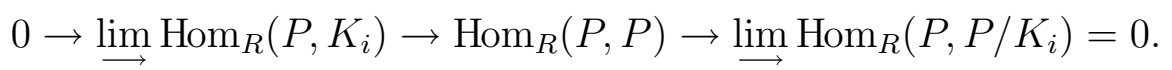

This implies that, for some $i \in I$, there exists $f \in \operatorname{Hom}_{R}\left(P, K_{i}\right)$ for which the composition $P \stackrel{f}{\rightarrow} K_{i} \rightarrow P$ is the identity map. So $P$ is finitely generated.

Finally we fix some notation.

\subsection{Canonical maps.}

The functorial morphisms related to a bimodule ${ }_{R} P_{S}$ are denoted by

$$
\begin{array}{ll}
\mu_{N}: P \otimes_{S} \operatorname{Hom}_{R}(P, N) \rightarrow N, & p \otimes f \mapsto(p) f, \\
\nu_{X}: X \rightarrow \operatorname{Hom}_{R}\left(P, P \otimes_{S} X\right), & x \mapsto[p \mapsto p \otimes x],
\end{array}
$$

where $N \in R$-Mod and $X \in S$-Mod.

\section{Projectivity conditions}

We now consider various projectivity properties. The basic relationships derived will be most helpful for the investigation of tilting modules.

Definitions. A module $P \in \sigma[M]$ is called $\sigma[M]$-projective if $P$ is projective in $\sigma[M]$;

$\operatorname{Gen}(M)$-projective if $\operatorname{Hom}_{R}(P,-)$ respects exact sequences in $\operatorname{Gen}(M)$; 
self-Ext-projective in $\sigma[M]$ if $\operatorname{Gen}(P) \subset P^{\perp_{M}}$;

$w$ - $\Sigma$-quasiprojective if $\operatorname{Hom}_{R}(P,-)$ respects exactness of sequences

$0 \rightarrow K \rightarrow P^{(\Lambda)} \rightarrow N \rightarrow 0$, where $K \in \operatorname{Gen}(P), \Lambda$ any set;

self-pseudo-projective in $\sigma[M]$ if any diagram with exact sequence

$$
\begin{aligned}
& \begin{array}{lll}
P & \stackrel{\alpha}{*} P
\end{array}
\end{aligned}
$$

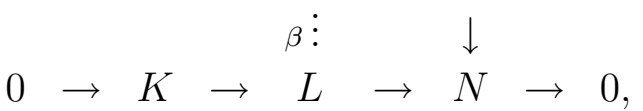

where $K \in \operatorname{Gen}(P)$ and $L \in \sigma[M]$, can be non-trivially commutatively extended by some $\alpha: P \rightarrow P, \beta: P \rightarrow L$.

Obviously we have the implications

$$
\sigma[P] \text {-projective } \Rightarrow \text { Gen }(P) \text {-projective } \Rightarrow \mathrm{w} \text { - } \sum \text {-quasiprojective. }
$$

By 3.3, $\operatorname{Gen}(P)$-projective is equivalent to self-Ext-projective in $\sigma[P]$ and it implies self-pseudo-projective in $\sigma[P]$

Recall that a finitely generated module $P$ is $\sigma[M]$-projective if and only if it is $M$ projective. $P$ is said to be minimal in $\operatorname{Gen}(P)$ if for any decomposition $P=P^{\prime} \oplus P^{\prime \prime}$, we have $\operatorname{Tr}\left(P^{\prime}, P^{\prime \prime}\right) \neq P^{\prime \prime}$.

The interest in self-pseudo-projective modules is motivated by the following observation (see $[20,3.4,3.8],[1, \S 1])$ :

3.1 Self-pseudo-projective modules in $\sigma[M]$.

Let $P \in \sigma[M]$ and $S=\operatorname{End}_{R}(P)$. The following are equivalent:

(a) $P$ is self-pseudo-projective in $\sigma[M]$;

(b) $\operatorname{Gen}(P)$ is closed under extensions in $\sigma[M]$.

Suppose that ${ }_{R} P$ is finitely generated and minimal, and $S$ is right perfect or $P_{S}$ is finitely generated and $S$ is semiperfect. Then (a)-(b) are equivalent to:

(c) $P$ is self-Ext-projective in $\sigma[M]$.

$\mathrm{w}$ - $\sum$-quasiprojective modules were introduced in Menini-Orsatti [15] to characterize $*$-modules. The following is a key result for the application of this notion.

\section{$3.2 \mathrm{w}$ - $\Sigma$-quasiprojective modules.}

(1) For a module $P$ the following are equivalent:

(a) $P$ is $w$ - $\Sigma$-quasiprojective;

(b) $\operatorname{Hom}_{R}(P,-)$ respects exact sequences $0 \rightarrow K \rightarrow L \rightarrow N \rightarrow 0$, where $K \in \operatorname{Gen}(P)$ and $L \in \operatorname{Pres}(P)$. 
(2) Let $P$ be $w$ - $\Sigma$-quasiprojective. Then:

(i) Factors of P-presented modules by P-generated submodules are P-presented.

(ii) For $N \in \operatorname{Pres}_{f}(P), \mu_{N}: P \otimes_{S} \operatorname{Hom}_{R}(P, N) \rightarrow N$ is an isomorphism.

(iii) If ${ }_{S} X$ is finitely generated then $\nu_{X}: X \rightarrow \operatorname{Hom}_{R}\left(P, P \otimes_{S} X\right)$ is epic.

(3) If $\operatorname{Gen}(P)=\operatorname{Pres}(P)$ the following are equivalent:

(a) $P$ is $w$ - $\Sigma$-quasiprojective;

(b) $P$ is $\operatorname{Gen}(P)$-projective.

Proof. (1) $(b) \Rightarrow(a)$ is trivial.

$(a) \Rightarrow(b)$ For any morphism $h: P \rightarrow N$, we have an exact commutative diagram

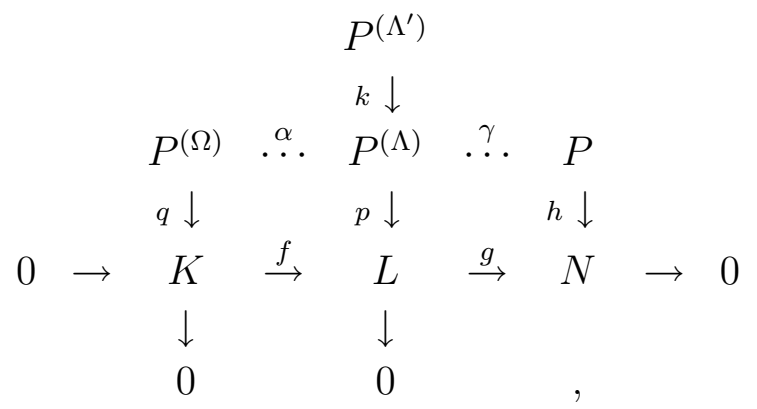

where $\Lambda, \Lambda^{\prime}$, and $\Omega$ are suitable sets.

By $(a)$, there exists $\alpha: P^{(\Omega)} \rightarrow P^{(\Lambda)}$ with $\alpha p=q f$. By construction, the kernel of

$$
p g: P^{(\Lambda)} \rightarrow N
$$

is equal to $\operatorname{Im} k+\operatorname{Im} \alpha$ and hence is $P$-generated. Again applying $(a)$, we obtain some $\gamma: P \rightarrow P^{(\Lambda)}$ with $\gamma p g=h$ proving our statement.

(2) (i) follows from the above proof, and (ii),(iii) from the proof of 5.1.

(3) follows immediately from (1).

Observation (3) implies Colpi [2, Corollary 4.2].

Next we investigate a projectivity property of $P$ with respect to $M$.

\subsection{Gen $(M)$-projective modules.}

For $P \in \sigma[M]$, the following are equivalent:

(a) $P$ is $\operatorname{Gen}(M)$-projective;

(b) $\operatorname{Hom}_{R}(P,-)$ respects exact sequences $0 \rightarrow K \rightarrow L \rightarrow N \rightarrow 0$, where $K \in \operatorname{Gen}(M)$ and $L \in \sigma[M]$;

(c) for each $K \in \operatorname{Gen}(M), \operatorname{Ext}_{M}^{1}(P, K)=0$ (i.e., $\left.\operatorname{Gen}(M) \subset P^{\perp_{M}}\right)$; 
(d) (i) $\operatorname{Ext}_{M}^{1}\left(P, M^{(\Lambda)}\right)=0$, for any set $\Lambda$;

(ii) $\operatorname{Ext}_{M}^{2}(P, N)=0$, for each $N \in \sigma[M]$.

Proof. $(a) \Rightarrow(b)$ Let $f: P \rightarrow N$ be any morphism. From the exact sequence in $(b)$ and the $M$-injective hull $i: L \rightarrow \widehat{L}$, we construct the commutative diagram,

$$
\begin{aligned}
& \text { P } \\
& 0 \rightarrow K \rightarrow L \stackrel{g}{\rightarrow} N \quad N \quad \rightarrow \quad 0
\end{aligned}
$$

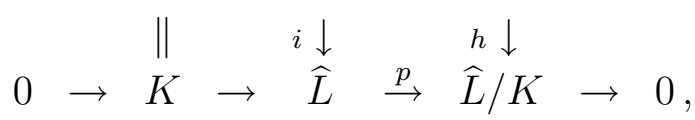

where the lower exact sequence is in $\operatorname{Gen}(M)$ and the right hand square is a pullback. By hypothesis there exists $\alpha: P \rightarrow \widehat{L}$ satisfying $\alpha p=f h$. Now the pullback property yields some $k: P \rightarrow L$ with

$$
f h=\alpha p=k i p=k g h .
$$

Since $h$ is mono we conclude $f=k g$. This proves our assertion.

$(b) \Rightarrow(a)$ and $(b) \Leftrightarrow(c)$ are obvious.

$(c) \Rightarrow(d)$ Clearly $\operatorname{Ext}_{M}^{1}\left(P, M^{(\Lambda)}\right)=0$, for any $\Lambda$.

For any $N \in \sigma[M]$, consider the exact sequence

$$
0 \rightarrow N \rightarrow \widehat{N} \rightarrow \widehat{N} / N \rightarrow 0
$$

where $\widehat{N}$ denotes the $M$-injective hull of $N$. From this we obtain the exact sequence

$$
0=\operatorname{Ext}_{M}^{1}(P, \widehat{N} / N) \rightarrow \operatorname{Ext}_{M}^{2}(P, N) \rightarrow \operatorname{Ext}_{M}^{2}(P, \widehat{N})=0,
$$

proving $\operatorname{Ext}_{M}^{2}(P, N)=0$.

$(d) \Rightarrow(c)$ By the connecting morphisms of the Ext-functor, (ii) implies that $P^{\perp_{M}}$ is closed under factor modules. Hence (i) implies $\operatorname{Gen}(M) \subset P^{\perp_{M}}$.

\section{Tilting modules}

Definition. We call $P \in \sigma[M]$ a tilting module in $\sigma[M]$ if

(i) $P$ is $\operatorname{Gen}(P)$-projective,

(ii) every $P$-generated module is $P$-presented (i.e., $\operatorname{Gen}(P)=\operatorname{Pres}(P)$ ),

(iii) $P$ is a subgenerator in $\sigma[M]$. 
$P$ is called self-tilting if it is tilting in $\sigma[P]$, i.e., if (i) and (ii) hold.

For a tilting module $P$ in $\sigma[M]$ we always have $\sigma[M]=\sigma[P]$ (by (iii)). So it is enough to study self-tilting modules in detail. Notice that we do not assume a tilting module to be finitely generated.

Clearly any projective generator in $\sigma[M]$ is tilting in $\sigma[M]$. The next proposition shows how close tilting modules are to projective generators.

4.1 Proposition. Let $P$ be a self-tilting module.

(1) If $P$ is a self-generator then $P$ is self-projective.

(2) The following are equivalent:

(a) $P$ is a generator in $\sigma[P]$;

(b) $P$ is projective in $\sigma[P]$.

Proof. (1) and (2) $(a) \Rightarrow(b)$ are obvious. $(b) \Rightarrow(a)$ will follow from $4.2(d)$, since for $P$ projective in $\sigma[P], \sigma[P]=P^{\perp_{P}}$.

Applying our knowledge about projectivity conditions from section 3 we obtain the following characterizations of

\subsection{Self-tilting modules.}

For a module $P$ the following are equivalent:

(a) $P$ is self-tilting;

(b) $\operatorname{Gen}(P)=\operatorname{Pres}(P)$ and $P$ is $w$ - $\Sigma$-quasiprojective;

(c) $\operatorname{Gen}(P)=\operatorname{Pres}(P)$ and $\operatorname{Gen}(P) \subset P^{\perp_{P}}$;

(d) $\operatorname{Gen}(P)=P^{\perp_{P}}$;

(e) (i) $\operatorname{Ext}_{P}^{1}\left(P, P^{(\Lambda)}\right)=0$, for any set $\Lambda$,

(ii) $\operatorname{Ext}_{P}^{2}(P, N)=0$, for all $N \in \sigma[P]$,

(iii) for $N \in \sigma[P], \operatorname{Hom}_{R}(P, N)=0=\operatorname{Ext}_{P}^{1}(P, N)=0$ implies $N=0$.

In case $\sigma[P]$ has a progenerator $G$, then (iii) is equivalent to

(iv) there exists an exact sequence

$$
0 \rightarrow G \rightarrow P_{1} \rightarrow P_{2} \rightarrow 0, \text { where } P_{1}, P_{2} \in \operatorname{Add}(P)
$$

If $P$ is self-tilting, then $\operatorname{Gen}(P)$ is closed under extensions and products in $\sigma[P]$. 
Proof. (1) $(a) \Leftrightarrow(b)$ and $(b) \Leftrightarrow(c)$ follow from 3.2 and 3.3, respectively.

$(c) \Rightarrow(d)$ Obviously $\operatorname{Gen}(P) \subset P^{\perp_{P}}$.

Let $N \in P^{\perp_{P}}$ and $N \rightarrow \widehat{N}$ the $P$-injective hull. With a $P$-representation of $\widehat{N} / N$, we obtain - by a pullback construction - the commutative exact diagram

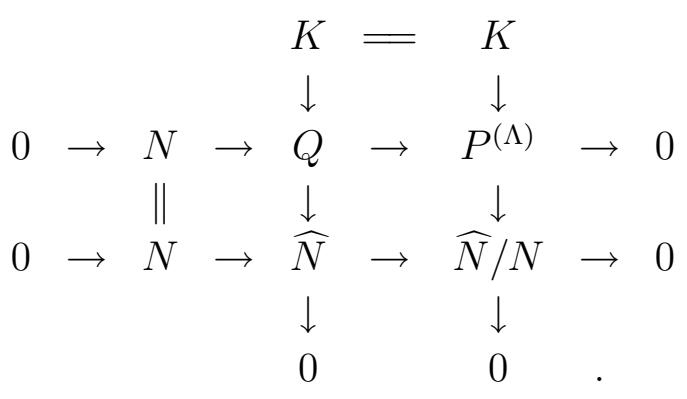

By 3.1, $\operatorname{Gen}(P)$ is closed under extensions in $\sigma[P]$ and hence $Q \in \operatorname{Gen}(P)$. Since $\operatorname{Ext}_{P}^{1}\left(P^{(\Lambda)}, N\right)=0$ the central sequence splits and so $N \in \operatorname{Gen}(P)$.

$(d) \Rightarrow(c)$ (see also [6, Lemma 1.2]) Let $N \in \operatorname{Gen}(P)$ and $\Lambda=\operatorname{Hom}_{R}(P, N)$. With the canonical sequence on the bottom and any extension on the top we have the diagram

$$
\begin{aligned}
& 0 \rightarrow K \rightarrow L \rightarrow P \rightarrow 0 \\
& \| \quad \alpha \vdots \quad \beta \text { : } \\
& 0 \rightarrow K \rightarrow P^{(\Lambda)} \rightarrow N \rightarrow 0
\end{aligned}
$$

which can be extended by some $\alpha: L \rightarrow P^{(\Lambda)}\left(\right.$ since $\left.\operatorname{Ext}_{P}^{1}\left(P, P^{(\Lambda)}\right)=0\right)$ and $\beta: P \rightarrow N$ commutatively. Since $P$ is projective with respect to the lower sequence we conclude (by the Homotopy Lemma) that the first sequence splits. Hence $\operatorname{Ext}_{P}^{1}(P, K)=0$ implying $K \in \operatorname{Gen}(P)$.

$(d) \Rightarrow(e)$ (i) and (ii) are shown in 3.3; (iii) is obvious.

$(e) \Rightarrow(d)$ By 3.3, $\operatorname{Gen}(P) \subset P^{\perp_{P}}$. Let $N \in P^{\perp_{P}}$ and put $\bar{N}=\operatorname{Tr}(P, N)$. By (ii), $P^{\perp_{P}}$ is closed under factor modules and so $N / \bar{N} \in P^{\perp_{P}}$. Moreover, since $\operatorname{Gen}(P)$ is closed under extensions in $\sigma[P]$ (by 3.3), $\operatorname{Hom}_{R}(P, N / \bar{N})=0$. Now (iii) implies $N / \bar{N}=0$ and so $N$ is $P$-generated.

(e) (iii) $\Rightarrow$ (iv) First we observe that $\operatorname{Gen}(P)=P^{\perp_{P}}$ implies that for any set $\Lambda$, $\left.P\right|_{P} ^{\Lambda}$ is $P$-generated and hence by $2.1, \operatorname{Hom}_{R}(G, P)_{S}$ is finitely generated, say by $\left\{f_{1}, \ldots, f_{k}\right\}$. Then the map

$$
i: G \rightarrow P^{k}, g \mapsto\left((g) f_{1}, \ldots,(g) f_{k}\right),
$$

is a monomorphism and $\operatorname{Hom}_{R}\left(P^{k}, P\right) \stackrel{\operatorname{Hom}(i, P)}{\rightarrow} \operatorname{Hom}_{R}(G, P)$ is epi. Hence $P$ is injective with respect to $i$ and so is $P^{n}$, for $n \in \mathbb{N}$. 
Consider the diagram

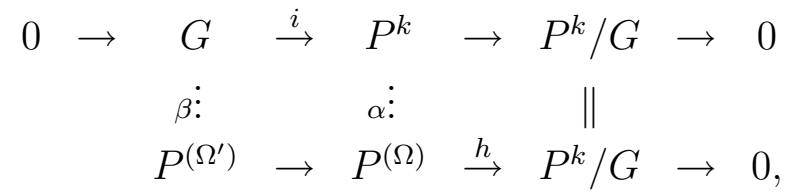

where the lower sequence is some $P$-presentation of $P^{k} / G$. By the given projectivity conditions there exist $\alpha: P^{k} \rightarrow P^{(\Omega)}$ and $\beta: G \rightarrow P^{(\Omega)}$ yielding a commutative diagram. Now $\operatorname{Im} \beta$ is contained in a finite summand of $P^{\left(\Omega^{\prime}\right)}$, and by the injectivity property just mentioned, there exists $\gamma: P^{k} \rightarrow P^{\left(\Omega^{\prime}\right)}$ with $i \gamma=\beta$. By the Homotopy Lemma we get some morphism $P^{k} / G \rightarrow P^{(\Omega)}$ which splits $h$ and hence $P^{k} / G \in$ $\operatorname{Add}(P)$.

(iv) $\Rightarrow\left(\right.$ iii) Let $N \in \sigma[P]$ be such that $\operatorname{Hom}_{R}(P, N)=0=\operatorname{Ext}_{P}^{1}(P, N)=0$. Applying $\operatorname{Hom}_{R}(-, N)$ to the sequence in (iv) yields the exact sequence

$$
0=\operatorname{Hom}_{R}\left(P_{1}, N\right) \rightarrow \operatorname{Hom}_{R}(G, N) \rightarrow \operatorname{Ext}_{R}^{1}\left(P_{2}, N\right)=0,
$$

and so $\operatorname{Hom}_{R}(G, N)=0$ and $N=0$.

It follows from the preceding arguments that $\operatorname{Gen}(P)$ is closed under extensions and products in $\sigma[P]$.

By definition, a module $P \in \sigma[M]$ is tilting in $\sigma[M]$ if it is self-tilting and $P$ is a subgenerator in $\sigma[M]$. Besides the general characterization of the latter condition in 2.3 there are additional properties of a self-tilting module $P$ which make it a subgenerator in $\sigma[M]$ and we give some of them in our next proposition.

\subsection{Tilting modules in $\sigma[M]$.}

For a module $P \in \sigma[M]$ with $S=\operatorname{End}_{R}(P)$, the following are equivalent:

(a) $P$ is tilting in $\sigma[M]$;

(b) $P$ is self-tilting and a subgenerator in $\sigma[M]$;

(c) $P$ is self-tilting, $P$ cogenerates a subgenerator of $\sigma[M]$, and $\operatorname{Gen}(P)$ is closed under products in $\sigma[M]$;

(d) $\operatorname{Gen}(P)=P^{\perp_{M}}$;

(e) (i) $\operatorname{Ext}_{M}^{1}\left(P, P^{(\Lambda)}\right)=0$, for any set $\Lambda$,

(ii) $\operatorname{Ext}_{M}^{2}(P, N)=0$, for all $N \in \sigma[M]$,

(iii) for $N \in \sigma[M], \operatorname{Hom}_{R}(P, N)=0=\operatorname{Ext}_{M}^{1}(P, N)=0$ implies $N=0$.

In case $\sigma[M]$ has a progenerator $G$, (iii) is equivalent to 
(iv) there exists an exact sequence

$$
0 \rightarrow G \rightarrow P_{1} \rightarrow P_{2} \rightarrow 0, \text { where } P_{1}, P_{2} \in \operatorname{Add}(P)
$$

Under these conditions $\operatorname{Hom}_{R}(G, P)_{S}$ is finitely generated.

Proof. $(a) \Leftrightarrow(b)$ is just the definition.

$(b) \Rightarrow(d),(e)$ is clear by 4.2 since $\sigma[P]=\sigma[M]$.

$(d) \Rightarrow(c)$ It follows from 4.2 that $\operatorname{Gen}(P)$ is closed under products. Since injective modules in $\sigma[M]$ are in $P^{\perp_{M}}$ they are $P$-generated and hence $P$ is a subgenerator in $\sigma[M]$.

$(c) \Rightarrow(b)$ Let $N$ be a subgenerator in $\sigma[M]$ which is cogenerated by $P$. Then $N$ is contained in a product of copies of $P$ in $\sigma[M]$, which is $P$-generated. This implies $\sigma[P]=\sigma[N]=\sigma[M]$.

$(e) \Rightarrow(d)$ We show that $\widehat{M}$, the $M$-injective hull of $M$, is $P$-generated. Then the assertion will follow from 4.2 .

Let $\bar{M}=\operatorname{Tr}(P, \widehat{M})$. (i), (ii) imply that $\widehat{M} / \bar{M} \in P^{\perp_{M}}$ and that $P$ is self-pseudoprojective in $\sigma[M]$, i.e., $\operatorname{Gen}(P)$ is closed under extensions in $\sigma[M]$. Therefore $\operatorname{Hom}_{R}(P, \widehat{M} / \bar{M})=0$ and $\widehat{M} / \bar{M}=0$ (by (iii)). So $\widehat{M} \in \operatorname{Gen}(P)$.

The final assertion follows from 2.1.

Putting $M=R=G$ the above theorem describes tilting modules in $R$-Mod. Notice that $P$ cogenerates a subgenerator in $R$-Mod if and only if $P$ is a faithful $R$-module and hence we have:

\subsection{Tilting modules in $R$-Mod.}

For an $R$-module $P$ with $S=\operatorname{End}_{R}(P)$, the following are equivalent:

(a) $P$ is tilting in $R$-Mod;

(b) $P$ is self-tilting and a subgenerator in $R$-Mod;

(c) $P$ is self-tilting, ${ }_{R} P$ is faithful and $P_{S}$ is finitely generated;

(d) $\operatorname{Gen}(P)=P^{\perp_{R}}$;

(e) (i) $\operatorname{Ext}_{M}^{1}\left(P, P^{(\Lambda)}\right)=0$, for any set $\Lambda$,

(ii) $\operatorname{Ext}_{M}^{2}(P, N)=0$, for all $N \in \sigma[M]$,

(iii) there exists an exact sequence

$$
0 \rightarrow R \rightarrow P_{1} \rightarrow P_{2} \rightarrow 0, \text { where } P_{1}, P_{2} \in \operatorname{Add}(P) .
$$

The conditions in 4.4(e) were used in Colpi-Trlifaj $[6,1.1]$ to define (not finitely generated) tilting modules in $R$-Mod and $(d) \Leftrightarrow(e)$ was shown in $[6,1.3]$. For ${ }_{R} P$ finitely generated, 4.4 corresponds to Colpi [3, Theorem 3]. 


\section{$5 \quad$ Self-small tilting modules}

A module $P$ is said to be self-small if, for any set $\Lambda$, the canonical map

$$
\operatorname{Hom}_{R}(P, P)^{(\Lambda)} \rightarrow \operatorname{Hom}_{R}\left(P, P^{(\Lambda)}\right)
$$

is an isomorphism. This implies that $\mu_{P^{(\Lambda)}}: P \otimes_{S} \operatorname{Hom}_{R}\left(P, P^{(\Lambda)}\right) \rightarrow P^{(\Lambda)}$ is also an isomorphism (where $S=\operatorname{End}_{R}(P)$ ).

Combined with projectivity conditions self-small modules have very interesting properties.

\subsection{Self-small w- $\Sigma$-quasiprojective modules.}

Let ${ }_{R} P$ be self-small and $w$ - $\Sigma$-quasiprojective and $S=\operatorname{End}_{R}(P)$. Then:

(1) For every P-presented module $N, \quad \mu_{N}: P \otimes_{S} \operatorname{Hom}_{R}(P, N) \rightarrow N$ is an isomorphism.

(2) For every left $S$-module $X, \nu_{X}: X \rightarrow \operatorname{Hom}_{R}\left(P, P \otimes_{S} X\right)$ is an epimorphism.

(3) $\operatorname{Pres}(P)$ is closed under direct limits.

(4) $\operatorname{Hom}_{R}(P,-)$ commutes with direct limits of P-presented modules.

Proof. (1) With a $P$-presentation of $N$ as lower sequence we have the commutative exact diagram

$$
\begin{array}{cccccc}
P \otimes_{S} \operatorname{Hom}_{R}(P, K) & \rightarrow P \otimes_{S} \operatorname{Hom}_{R}\left(P, P^{(\Lambda)}\right) & \rightarrow & P \otimes_{S} \operatorname{Hom}_{R}(P, N) & \rightarrow 0 \\
\mu_{K} \downarrow & & \simeq \downarrow & & \downarrow \mu_{N} & \\
K & \rightarrow & P^{(\Lambda)} & \rightarrow & N & \rightarrow 0,
\end{array}
$$

where $\mu_{K}$ is epi ( $K$ is $P$-generated) and hence $\mu_{N}$ is an isomorphism.

(2) Let $S^{(\Omega)} \rightarrow S^{(\Lambda)} \rightarrow X \rightarrow 0$ be an $S$-presentation of $X$. Tensoring with $P_{S}$ yields an exact sequence

$$
0 \rightarrow U \rightarrow P \otimes_{S} S^{(\Lambda)} \rightarrow P \otimes_{S} X \rightarrow 0
$$

where $U$ is $P$-generated. Applying $\operatorname{Hom}_{R}(P,-)$ we obtain the commutative exact diagram

$$
\begin{aligned}
& \begin{array}{lll}
S^{(\Lambda)} & \rightarrow & X
\end{array} \quad \rightarrow 0 \\
& \operatorname{Hom}_{R}\left(P, P \otimes_{S} S^{(\Lambda)}\right) \rightarrow \operatorname{Hom}_{R}\left(P, P \otimes_{S} X\right) \rightarrow 0,
\end{aligned}
$$

showing that $\nu_{X}$ is epi. 
(3) For any family $\left\{N_{\lambda}\right\}_{\Lambda}$ of $P$-presented modules, the map

$$
\begin{aligned}
\nu: \bigoplus_{\Lambda} \operatorname{Hom}_{R}\left(P, N_{\lambda}\right) \rightarrow \operatorname{Hom}_{R}\left(P, P \otimes_{S} \bigoplus_{\Lambda} \operatorname{Hom}_{R}\left(P, N_{\lambda}\right)\right) \\
\simeq \operatorname{Hom}_{R}\left(P, \oplus_{\Lambda} N_{\lambda}\right),
\end{aligned}
$$

is injective (on any finite partial sum) and is epic by (1). The isomorphism on the right hand side follows from $(2)$. This proves that $\operatorname{Hom}_{R}(P,-)$ commutes with direct sums in $\operatorname{Pres}(P)$.

Now let $\left\{N_{i}, f_{i j}\right\}_{I}$ be a direct system of $P$-presented modules. Putting $N_{i, j}=N_{i}$, for any $i \leq j$, the direct limit is defined by an exact sequence of the form (see [19, 24.2])

$$
\bigoplus_{i \leq j} N_{i, j} \rightarrow \bigoplus_{I} N_{i} \rightarrow \underset{\lim }{\longrightarrow} N_{i} \rightarrow 0
$$

By $3.2, \lim N_{i}$ is $P$-presented.

(4) In a canonical way, $\left\{\operatorname{Hom}_{R}\left(P, N_{i}\right), \operatorname{Hom}_{R}\left(P, f_{i j}\right)\right\}_{I}$ is a directed family of $S$ modules and we have a pure exact sequence in $S$-Mod (e.g., $[19,33.9]$ ),

$$
0 \rightarrow X \rightarrow \bigoplus_{I} \operatorname{Hom}_{R}\left(P, N_{i}\right) \rightarrow \underset{\lim }{\longrightarrow} \operatorname{Hom}_{R}\left(P, N_{i}\right) \rightarrow 0
$$

Since $P \otimes_{S}-$ preserves exactness of this sequence and commutes with direct limits we obtain a short exact sequence (with the isomorphism from 2.5(2))

$$
0 \rightarrow P \otimes_{S} X \rightarrow \bigoplus_{I} N_{i} \rightarrow \underset{\lim }{\longrightarrow} N_{i} \rightarrow 0
$$

$\operatorname{Hom}_{R}(P,-)$ is exact on this sequence and we get the commutative exact diagram

$$
\begin{aligned}
& 0 \rightarrow \quad X \quad \rightarrow \bigoplus_{I} \operatorname{Hom}_{R}\left(P, N_{i}\right) \rightarrow \underset{\sim}{\longrightarrow} \lim _{R}\left(P, N_{i}\right) \rightarrow 0 \\
& \nu_{X} \downarrow \quad \simeq \downarrow \quad \alpha \downarrow \\
& 0 \rightarrow \operatorname{Hom}_{R}\left(P, P \otimes_{S} X\right) \rightarrow \operatorname{Hom}_{R}\left(P, \oplus_{I} N_{i}\right) \rightarrow \operatorname{Hom}_{R}\left(P, \underset{\lim }{\longrightarrow} N_{i}\right) \rightarrow 0 .
\end{aligned}
$$

Since $\nu_{X}$ is epi (by 5.1(2)) we conclude that $\alpha$ is an isomorphism.

Self-small tilting modules in $\sigma[M]$ and $R$-Mod can be described by the characterization of subgenerators (in 2.3) and of tilting modules (in 4.3,4.4). There are some more properties which are worth mentioning.

\subsection{Self-small self-tilting modules.}

For an $R$-module $P$ the following are equivalent:

(a) $P$ is self-small and self-tilting;

(b) (i) $P$ is finitely presented in $\sigma[P]$ and $\operatorname{Ext}_{P}^{1}(P, P)=0$,

(ii) $\operatorname{Ext}_{P}^{2}(P, N)=0$, for all $N \in \sigma[P]$, 
(iii) for $N \in \sigma[P], \operatorname{Hom}_{R}(P, N)=0=\operatorname{Ext}_{P}^{1}(P, N)=0$ implies $N=0$.

If $\sigma[P]$ has a progenerator $G$, then (iii) is equivalent to

(iv) there exists an exact sequence

$$
0 \rightarrow G \rightarrow P_{1} \rightarrow P_{2} \rightarrow 0, \text { where } P_{1}, P_{2} \in \operatorname{add}(P)
$$

Under these conditions $\operatorname{Hom}_{R}(G, P)_{S}$ is finitely presented in Mod-S.

Proof. $\quad(a) \Rightarrow(b)$ By 5.1, $\operatorname{Hom}_{R}(P,-)$ commutes with direct limits in $\operatorname{Gen}(P)(=$ $\operatorname{Pres}(P)$ ) and hence $P$ is finitely presented in $\sigma[P]$ (by 2.4). The remaining assertions follow from 4.2. Since ${ }_{R} P$ is finitely generated we have $P_{1}, P_{2} \in$ add $(P)$.

$(b) \Rightarrow(a)$ By 2.4(2), $\operatorname{Ext}_{R}^{1}(P, P)=0$ implies $\operatorname{Ext}_{R}^{1}\left(P, P^{(\Lambda)}\right)=0$.

For any $\Lambda,\left.P\right|_{P} ^{\Lambda}$ is $P$-presented and by 5.1 we have

$$
\left.P \otimes_{S} S^{\Lambda} \simeq P \otimes_{S} \operatorname{Hom}_{R}\left(P, P^{\Lambda}\right) \simeq P\right|_{P} ^{\Lambda}
$$

Since $G$ is a progenerator in $\sigma[P]$, this implies

$$
\operatorname{Hom}_{R}(G, P) \otimes_{S} S^{\Lambda} \simeq \operatorname{Hom}_{R}\left(G, P \otimes_{S} S^{\Lambda}\right) \simeq \operatorname{Hom}_{R}\left(G,\left.P\right|_{P} ^{\Lambda}\right) \simeq \operatorname{Hom}_{R}(G, P)^{\Lambda} .
$$

By $[19,12.9]$ this is equivalent to $\operatorname{Hom}_{R}(G, P)_{S}$ being finitely presented.

Specializing to $\sigma[P]=R$-Mod we obtain:

\subsection{Self-small tilting modules in $R$-Mod.}

For a self-small $R$-module $P$ with $S=\operatorname{End}_{R}(P)$, the following are equivalent:

(a) $P$ is tilting in $R$-Mod;

(b) ${ }_{R} P$ is faithful and self-tilting and $\operatorname{Gen}(P)$ is closed under products in $R$-Mod;

(c) ${ }_{R} P$ is faithful and self-tilting and $P_{S}$ is finitely generated (finitely presented);

(d) (i) ${ }_{R} P$ is finitely presented and $\operatorname{Ext}_{R}^{1}(P, P)=0$,

(ii) $\operatorname{Ext}_{R}^{2}(P, N)=0$, for all $N \in R$-Mod (i.e. proj.dim $(P) \leq 1$ ),

(iii) there exists a short exact sequence

$$
0 \rightarrow R \rightarrow P_{1} \rightarrow P_{2} \rightarrow 0, \text { where } P_{1}, P_{2} \in \text { add }(P)
$$

Proof. $(a) \Leftrightarrow(b)$ follows from $4.3,(c) \Rightarrow(a)$ from 2.3.

$(a) \Rightarrow(c)$ and $(c) \Leftrightarrow(d)$ follow from 5.2.

Notice that 5.3 yields the characterization of the classical tilting modules we mentioned in the introduction. 
5.4 Remarks. *-modules are characterized as self-small $\mathrm{w}$ - $\Sigma$-quasiprojective modules $P$ for which $\operatorname{Gen}(P)=\operatorname{Pres}(P)$ (e.g., Colpi [2, Theorem 4.1]). In view of 3.2 they are just the self-small self-tilting modules. The characterization given in 5.2 was obtained in Folz [11, 3.12].

Combining 2.5, 5.1, 2.3, and 4.3 we obtain characterizations of $*$-modules and finitely generated tilting modules in $R$-Mod as given in Menini-Orsatti [5, Theorem 4.3], Colpi [2, Proposition 1.5] and [3, Theorems 3 and 5]. It was shown in Trlifaj [17] that such modules are finitely generated. In Colpi-Menini [5, Proposition 1.7] it is proved that finitely generated tilting modules in $R$-Mod are finitely presented in $R$-Mod. Both results are subsumed in 5.2.

In the recent preprint Colpi [4] alternative proofs can be found for $2.1,4.2,(\mathrm{~d}) \Leftrightarrow(\mathrm{e})$, and 5.2.

The isomorphisms obtained in 5.1 indicate the existence of equivalences between the categories $\operatorname{Pres}(P)$ or $\operatorname{Gen}(P)$ and certain subcategories of $S$-Mod. To describe these let $Q$ be any cogenerator in $\sigma[P]$ and put $U=\operatorname{Hom}_{R}(P, Q)$. Then we have for any $X \in S$-Mod,

$$
\operatorname{Ke} \nu_{X}=\left\{x \in X \mid P \otimes_{S} S x=0\right\}=\operatorname{Re}(X, U) .
$$

This can be easily seen from the canonical isomorphism

$$
\operatorname{Hom}_{S}(X, U)=\operatorname{Hom}_{S}\left(X, \operatorname{Hom}_{R}(P, Q)\right) \simeq \operatorname{Hom}_{R}\left(P \otimes_{S} X, Q\right),
$$

using the cogenerator property of $Q$.

Let $\operatorname{Kog}\left({ }_{S} U\right)$ denote the full subcategory of $S$-Mod determined by all modules which are cogenerated by $U$, and $\operatorname{Kog}_{f}\left({ }_{S} U\right)$ the class of finitely generated modules in $\operatorname{Kog}\left({ }_{S} U\right)$. By 5.1, for any self-small self-tilting module $P, \nu_{X}$ is an isomorphism for all objects in $\operatorname{Kog}\left({ }_{S} U\right)$ and clearly

$$
\operatorname{Kog}\left({ }_{S} U\right)=\left\{X \in S-M o d \mid X \simeq \operatorname{Hom}_{R}(P, N), N \in \operatorname{Gen}(P)\right\} .
$$

This yields the following

\subsection{Equivalences.}

For an $R$-module $P$ with $S=\operatorname{End}_{R}(P)$, let $Q$ be a cogenerator in $\sigma[P]$ and $U=\operatorname{Hom}_{R}(P, Q)$.

(1) If $P$ is $w$ - $\Sigma$-quasiprojective we have an equivalence

$$
\operatorname{Hom}_{R}(P,-): \operatorname{Pres}_{f}(P) \rightarrow \operatorname{Kog}_{f}\left({ }_{S} U\right) .
$$

(2) If $P$ is self-small and $w$ - $\Sigma$-quasiprojective we have an equivalence

$$
\operatorname{Hom}_{R}(P,-): \operatorname{Pres}(P) \rightarrow \operatorname{Kog}\left({ }_{S} U\right) .
$$


(3) If $P$ is self-small and self-tilting we have an equivalence

$$
\operatorname{Hom}_{R}(P,-): \operatorname{Gen}(P) \rightarrow \operatorname{Kog}\left({ }_{S} U\right) .
$$

In each case the inverse functor is $P \otimes_{S}-$.

Proof. (1) By 3.2 and 5.1 we have natural isomorphisms

$$
\mu: P \otimes_{S} \operatorname{Hom}_{R}(P,-) \rightarrow i d_{\operatorname{Pres}_{f}(P)}, \quad \nu: i d_{\operatorname{Kog}_{f}\left({ }_{S} U\right)} \rightarrow \operatorname{Hom}_{R}\left(P, P \otimes_{S}-\right) .
$$

From these the assertions follow.

(2) and (3) are shown by similar arguments.

Remarks. Observation (1) in 5.5 generalizes the fact that any self-projective module defines an equivalence between finitely $P$-presented $R$-modules and finitely presented $S$-modules (e.g., [19, 46.11]).

The equivalence in (2) was described in [2, Proposition 3.7].

(3) is essentially the Brenner-Butler Theorem (e.g., [1, Theorem 3.7]). It was noticed in Menini-Orsatti [15] and Colpi [2] that equivalences as described in (3) are induced by self-small self-tilting modules ( $=*$-modules).

5.6 Corollary. Let $P$ be a self-tilting $R$-module $P$ with $S=\operatorname{End}_{R}(P)$. Then $P$ is self-small if and only if $\operatorname{Im}_{\operatorname{Hom}_{R}}(P,-)$ is closed under $S$-submodules.

Proof. If $P$ is self-small the assertion follows from 5.5(4).

Assume that $\operatorname{Im~}_{\operatorname{Hom}_{R}}(P,-)$ is closed under submodules. Clearly for every set $\Lambda, S^{\Lambda} \in \operatorname{Im}_{\operatorname{Hom}_{R}}(P,-)$. If $\operatorname{Im} \operatorname{Hom}_{R}(P,-)$ is closed under submodules, this implies $S^{(\Lambda)} \in \operatorname{Im}_{\operatorname{Hom}_{R}}(P,-)$ and hence

$$
\operatorname{Hom}_{R}(P, P)^{(\Lambda)} \simeq S^{(\Lambda)} \simeq \operatorname{Hom}_{R}\left(P, P \otimes_{S} S^{(\Lambda)}\right) \simeq \operatorname{Hom}_{R}\left(P, P^{\Lambda}\right),
$$

showing that $P$ is self-small.

Examples of self-small tilting modules are abundant in representation theory (e.g., [1], [14]). The following example of a not finitely generated tilting module is due to Fuchs [12] and was further investigated in Facchini [9, 10]. More examples of this type will be considered in the subsequent sections.

\subsection{Divisible modules over integral domains.}

Let $R$ be a commutative integral domain. Then the divisible $R$-module $\partial$ introduced in Fuchs [12], § 3, is tilting in R-Mod.

Proof. As shown in $[12, \S 3]$, the $\partial$-generated modules are precisely the divisible $R$-modules. By [12, Proposition 11], $\operatorname{Ext}_{R}^{n}(\partial, D)=0$, for all divisible $R$-modules $D$ and for all $n \geq 1$. By [12, Lemma 14], every $\partial$-generated module is $\partial$-presented and hence $\partial$ is tilting in $R$-Mod. 


\section{6 (Semi-) cohereditary modules}

As in the classical situation hereditary modules provide interesting examples for tilting modules. Since there may be no projectives in our categories we refer to the characterization of hereditary modules by injectives.

Let $\operatorname{Inj}(M)$ denote the class of all injectives, and w-Inj $(M)$ the class of all weakly $M$-injectives in $\sigma[M]$. For any subgenerator $P \in \sigma[M]$, we have $\operatorname{Inj}(M)=\operatorname{Inj}(P)$ and (by $[19,16.11]$ )

$$
\operatorname{Inj}(M) \subset \mathrm{w}-\operatorname{Inj}(M) \subset \operatorname{Gen}(P) .
$$

Moreover, $\operatorname{Inj}(M)=\mathrm{w}-\operatorname{Inj}(M)$ if and only if $M$ is locally noetherian.

In case $M$ is a submodule of a direct sum of finitely presented modules in $\sigma[M]$, any module in $\sigma[M]$ is weakly $M$-injective if and only if it is absolutely pure in $\sigma[M]$ $([19,35.4])$.

Recall that $P \in \sigma[M]$ is locally coherent in $\sigma[M]$ if every finitely generated submodule of $P$ is finitely presented in $\sigma[M]$, and $P$ is (semi-) hereditary in $\sigma[M]$ if every (finitely generated) submodule of $P$ is projective in $\sigma[M]$.

We call a module $P \in \sigma[M]$ (semi-) cohereditary in $\sigma[M]$ if every factor module of $P$ in (weakly) $M$-injective.

The following technical properties will be needed.

6.1 Proposition. Let $M$ be an $R$-module.

(1) $M$ is locally noetherian if and only if $\operatorname{Inj}(M)$ is closed under direct limits.

(2) If $M$ is locally coherent then w-Inj $(M)$ is closed under direct limits.

(3) Suppose that $M$ is a submodule of a direct sum of finitely presented modules and $\mathrm{w}-\operatorname{Inj}(M)$ is closed under direct limits. Then $M$ is locally coherent.

Proof. (1) is shown in [19, 27.3].

(2) Assume $M$ to be locally coherent and let $\left\{P_{i}\right\}_{I}$ be a direct family of weakly $M$-injective modules. For every finitely generated $K \subset M$ and $f: K \rightarrow \underline{\lim } P_{i}$, we have an exact diagram

$$
\begin{aligned}
& 0 \rightarrow \begin{array}{l}
K \\
\downarrow_{f}
\end{array} \quad \rightarrow \quad M \\
& \bigoplus_{I} P_{i} \rightarrow \underline{\lim } P_{i} \rightarrow 0
\end{aligned}
$$

Since the lower sequence is pure and $K$ is finitely presented, there exists some morphism $g: K \rightarrow \bigoplus_{I} P_{i}$ and - by the weak injectivity property of $\bigoplus_{I} P_{i}$ - some morphism $h: M \rightarrow \bigoplus_{I} P_{i}$ yielding a commutative diagram. This proves that $\lim _{i}$ is weakly $M$-injective.

(3) Now assume that $M$ is a submodule of a direct sum of finitely presented modules and w-Inj $(M)$ is closed under direct limits. 
Every finitely generated submodule $K \subset M$ is contained in some finitely presented module $L \in \sigma[M]$. For any direct family $\left\{P_{i}\right\}_{I}$ of weakly $M$-injective modules, we have the commutative exact diagram

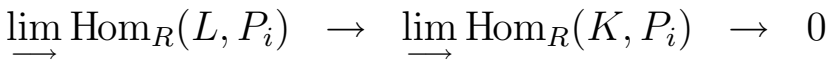

$$
\begin{aligned}
& \downarrow \Phi_{L} \quad \downarrow \Phi_{K} \\
& \operatorname{Hom}_{R}\left(L, \lim _{\longrightarrow} P_{i}\right) \rightarrow \operatorname{Hom}_{R}\left(K, \underset{\lim }{\longrightarrow} P_{i}\right) \rightarrow 0
\end{aligned}
$$

where $\Phi_{L}$ is an isomorphism and $\Phi_{K}$ is mono. From the diagram we conclude that $\Phi_{K}$ is also epi and so $\operatorname{Hom}_{R}(K,-)$ commutes with direct limits of weakly $M$-injective modules. So $K$ is finitely presented in $\sigma[M]$ (by 2.4) and $M$ is locally coherent.

Next we list some elementary statements about (semi-) cohereditary modules.

6.2 Proposition. Let $M$ be an $R$-module.

(1) If $P_{1}, \ldots, P_{k}$ are cohereditary in $\sigma[M]$ then so is $\bigoplus_{i=1}^{k} P_{i}$.

(2) If $M$ is locally noetherian then any direct sum of cohereditary modules in $\sigma[M]$ is cohereditary in $\sigma[M]$.

(3) Let $M$ be a submodule of a direct sum of finitely presented modules in $\sigma[M]$.

(i) Any finite direct sum of semi-cohereditary modules is semi-cohereditary.

(ii) If $M$ is locally coherent in $\sigma[M]$, then any direct sum of semi-cohereditary modules is semi-cohereditary.

Proof. (1) Let $P_{1}, P_{2}$ be cohereditary in $\sigma[M]$ and $P=P_{1} \oplus P_{2}$. For any submodule $K \subset P, P_{1} /\left(K \cap P_{1}\right)$ is $M$-injective and we have

$$
P / K \simeq P_{1} /\left(K \cap P_{1}\right) \oplus \bar{P}_{2},
$$

where $\bar{P}_{2}$ is some factor module of $P_{2}$ and hence is also $M$-injective. So $P / K$ is $M$-injective and $P$ is cohereditary.

For finite direct sums the assertation follows by induction.

(2) Let $\left\{P_{\lambda}\right\}_{\Lambda}$ be a family of cohereditary modules in $\sigma[M]$ and $K \subset \bigoplus_{\Lambda} P_{\lambda}$. Now $\bigoplus_{\Lambda} P_{\lambda}$ is the direct limit of its finite partial sums, and hence $\bigoplus_{\Lambda} P_{\lambda} / K$ is the direct limit of $M$-injective modules (by (1)) which is $M$-injective (by 6.1).

(3) As noticed above the weakly $M$-injectives are just the absolutely pure modules in $\sigma[M]$.

(i) Let $P_{1}, P_{2}$ be semi-cohereditary in $\sigma[M]$ and $P=P_{1} \oplus P_{2}$. For any submodule $K \subset P$, we have a short exact sequence

$$
0 \rightarrow P_{1} /\left(K \cap P_{1}\right) \rightarrow P / K \rightarrow \bar{P}_{2} \rightarrow 0
$$


where $\bar{P}_{2}$ is some factor module of $P_{2}$ and so $\bar{P}_{2}$ and $P_{1} /\left(K \cap P_{1}\right)$ both are absolutely pure in $\sigma[M]$. By $[19,35.2]$, this implies that $P / K$ is absolutely pure in $\sigma[M]$ and so $P$ is semi-cohereditary. Now the assertion follows by induction.

(ii) In view of 6.1 the proof of (2) applies.

\subsection{Cohereditary modules.}

(1) For an $M$-injective, locally noetherian subgenerator $P \in \sigma[M]$, the following are equivalent:

(a) $P$ is cohereditary in $\sigma[M]$;

(b) every injective module is cohereditary in $\sigma[M]$;

(c) every indecomposable injective module is cohereditary in $\sigma[M]$.

In case $\sigma[M]$ has a projective subgenerator $L,(\mathrm{a})-(\mathrm{c})$ are equivalent to:

(d) $L$ is hereditary in $\sigma[M]$.

(2) For a subgenerator $P \in \sigma[M]$, the following are equivalent:

(a) $\operatorname{Gen}(P)=\operatorname{Inj}(M)$;

(b) $P$ is locally noetherian and cohereditary in $\sigma[M]$.

Proof. (1) $(a) \Leftrightarrow(b) \Rightarrow(c)$ follow from $6.2 ;(a) \Leftrightarrow(d)$ is shown in [19, 39.9].

$(c) \Rightarrow(b)$ This follows again by 6.2 since $P$ is a direct sum of indecomposables (by Matlis' Theorem).

(2) $(a) \Rightarrow(b)$ By $\operatorname{Gen}(P)=\operatorname{Inj}(M)$, factor modules and direct sums of copies of $P$ are $M$-injective and so $P$ is cohereditary and locally noetherian (by $[19,27.3]$ ).

$(b) \Rightarrow(a)$ Since $P$ is a subgenerator in $\sigma[M], \operatorname{Gen}(P) \supset \operatorname{Inj}(M)$. By $(1), P^{(\Lambda)}$ is cohereditary for any $\Lambda$, implying $\operatorname{Gen}(P) \subset \operatorname{Inj}(M)$.

\subsection{Semi-cohereditary modules.}

(1) For a weakly $M$-injective, locally coherent subgenerator $P \in \sigma[M]$, the following are equivalent:

(a) $P$ is semi-cohereditary in $\sigma[M]$;

(b) every weakly $M$-injective module is semi-cohereditary in $\sigma[M]$.

In case $\sigma[M]$ has a projective subgenerator $L,(\mathrm{a})-(\mathrm{c})$ are equivalent to:

(d) $L$ is semi-hereditary in $\sigma[M]$.

(2) For a module $P$, which is a submodule of a direct sum of finitely presented modules in $\sigma[P]$, the following are equivalent: 
(a) $\operatorname{Gen}(P)=\mathrm{w}-\operatorname{Inj}(P)$;

(b) $P$ is locally coherent and cohereditary in $\sigma[M]$.

Proof. (1) $(a) \Leftrightarrow(b)$ follows from $6.2 ;(b) \Leftrightarrow(c)$ is shown in $[19,39.5]$.

(2) Apply 6.1 and 6.2(3).

Next we show that (semi-) cohereditary modules and rings provide non-trivial examples of tilting modules.

\subsection{Cohereditary and tilting modules.}

(1) For $P \in \sigma[M]$ the following are equivalent:

(a) $P$ is tilting in $\sigma[M]$ and $\operatorname{Gen}(P)=\operatorname{Inj}(M)$;

(b) $\operatorname{Inj}(M)=\operatorname{Gen}(P)=P^{\perp_{M}}$;

(c) $\operatorname{Pres}(P)=\operatorname{Gen}(P)=\operatorname{Inj}(M)$;

(d) $P$ is locally noetherian and cohereditary and every injective module in $\sigma[M]$ is embedded in some $P^{(\Lambda)}$;

(e) $P$ is locally noetherian and cohereditary and any indecomposable $M$ injective module in $\sigma[M]$ is embedded in $P$.

(2) Let $M$ be locally noetherian and assume its $M$-injective hull $\widehat{M}$ to be cohereditary in $\sigma[M]$,

(i) If $\left\{U_{\omega}\right\}_{\Omega}$ is a representing family of indecomposable injectives in $\sigma[M]$, then $U=\bigoplus_{\Omega} U_{\omega}$ is tilting in $\sigma[M]$.

(ii) If there exists a progenerator $G \in \sigma[M]$ and $\widehat{G}$ denotes its $M$-injective hull, then $P:=\widehat{G} \oplus \widehat{G} / G$ is a tilting module in $\sigma[M]$.

Proof. (1) $(a) \Leftrightarrow(b) \Rightarrow(c)$ is clear by definition.

$(c) \Rightarrow(d)$ By 6.3, $P$ is locally noetherian and cohereditary. Now $\operatorname{Gen}(P)=$ $\operatorname{Pres}(P)$ implies that every $P$-generated module is contained in some direct sum $P^{(\Lambda)}$.

$(d) \Rightarrow(e)$ Every uniform submodule of $P^{(\Lambda)}$ embeds in $P$ (e.g., [13, p. 43]).

$(e) \Rightarrow(a)$ Clearly $\operatorname{Inj}(M)=\operatorname{Gen}(P)$ (by 6.3) and $P$ is $\operatorname{Gen}(P)$-projective since all short exact sequences in $\operatorname{Gen}(P)$ split. Any $P$-generated module $N$ is $M$-injective and so is a direct sum of indecomposable injective modules $(\subset P)$. Therefore $N \subset P^{(\Lambda)}$ for some $\Lambda$, and $N$ is in fact a direct summand, i.e., $N \in \operatorname{Pres}(P)$.

(2) (i) By 6.3, $U$ is cohereditary in $\sigma[M]$. So $U$ is tilting by (1)(e).

(ii) By definition of $P$, there exists an exact sequence

$$
0 \rightarrow G \rightarrow P_{1} \rightarrow P_{2} \rightarrow 0 \text {, where } P_{1}, P_{2} \in \operatorname{Add}(P) \text {. }
$$

Now the assertion follows from 4.3. 
Putting $M=R=G$ we obtain the

6.6 Corollary. For a ring $R$ with $R$-injective hull $E(R)$, let $\left\{U_{\omega}\right\}_{\Omega}$ be a minimal representing family of indecomposable injectives in $R$-Mod and $U=\bigoplus_{\Omega} U_{\omega}$.

If $R$ is noetherian and left hereditary then $U$ and $E(R) \oplus E(R) / R$ are tilting modules in $R$-Mod and $E(R) \oplus E(R) / R$ contains all the $U_{\omega}$ 's (as direct summands).

In particular, $Q \oplus \mathbb{Q} / \mathbb{Z}$ is a tilting module in $\mathbb{Z}$-Mod.

Note that by the above examples, for a noetherian hereditary ring the minimal injective cogenerator need not be tilting (in fact it is not a subgenerator) in $R$-Mod. It was observed by Colpi-Menini [5, Theorem 3.3] that the existence of a finitely generated tilting module $P \in R$-Mod with $\operatorname{Gen}(P)=\operatorname{Inj}(R)$ implies that $R$ is left artinian and hereditary (since by 6.5 the indecomposable injectives are finitely generated). For such rings the minimal injective cogenerator is clearly tilting in $R$-Mod. The following proposition generalizes this situation.

\subsection{Cogenerators as tilting modules.}

Let $Q$ denote the minimal injective cogenerator of $\sigma[M]$. Then the following are equivalent:

(a) There exists a tilting $P \in \sigma[M]$ with essential socle and $\operatorname{Gen}(P)=\operatorname{Inj}(M)$;

(b) $M$ is locally of finite length, and there exists a cohereditary cogenerator in $\sigma[M]$;

(c) $Q$ is locally of finite length and a cohereditary subgenerator in $\sigma[M]$;

(d) $Q$ is tilting in $\sigma[M]$ and $\operatorname{Gen}(Q)=\operatorname{Inj}(M)$.

Proof. $(a) \Rightarrow(b)$ By $6.5, P$ is locally noetherian and cohereditary. Moreover every $P$-generated module is contained in some direct sum $P^{(\Lambda)}$, which is a direct sum of $M$-injective hulls of simple modules.

Let $K \subset L \subset P$ be submodules with $L$ finitely generated. Then $L / K$ is embedded in a finite direct sum of injective hulls of simple modules and hence is finitely cogenerated. So $L$ is artinian (e.g., $[19,31.1])$.

$(b) \Rightarrow(c)$ Under the given conditions every cogenerator is a subgenerator in $\sigma[M]$ and $Q$ is cohereditary by 6.3 .

$(c) \Rightarrow(d)$ By $6.5(\mathrm{e}), Q$ is tilting in $\sigma[M]$; by $6.3(2), \operatorname{Gen}(Q)=\operatorname{Inj}(M)$.

$(d) \Rightarrow(a)$ is trivial.

The preceding observations yield a number of examples for tilting modules. As special cases we notice: 


\section{8 $Q / \mathbb{Z}$ and $\mathbb{Z}_{p^{\infty}}$ are self-tilting.}

The $\mathbb{Z}$-module $Q / \mathbb{Z}$ is tilting in $\sigma[Q / \mathbb{Z}]$, the category of torsion $\mathbb{Z}$-modules.

For any prime $p \in \mathbb{N}$, the $\mathbb{Z}$-module $\mathbb{Z}_{p^{\infty}}$ is tilting in $\sigma\left[\mathbb{Z}_{p^{\infty}}\right]$, the category of p-torsion $\mathbb{Z}$-modules.

As noticed previously (see 4.1) tilting modules are close to projective generators. The above example gives a tilting module in a category without projectives (see [19, 18.12]). We can generalize these considerations in the following way.

\subsection{Semi-cohereditary and self-tilting modules.}

For an $R$-module $P$ the following are equivalent:

(a) $P$ is self-tilting and $\operatorname{Gen}(P)=\mathrm{w}-\operatorname{Inj}(P)$;

(b) $\operatorname{Gen}(P) \subset P^{\perp_{P}}$ and for any finitely generated submodule $K \subset P^{k}$, where $k \in \mathbb{N}$, we have $P^{k} / K \in \operatorname{Add}(P)$.

If $P$ is a direct summand of a direct sum of finitely presented modules in $\sigma[P]$, then (a)-(c) are equivalent to:

(c) $P$ is locally coherent and semi-cohereditary, and for any finitely generated submodule $K \subset P^{k}, k \in \mathbb{N}$, we have $P^{k} / K \in \operatorname{Add}(P)$.

If $P$ satisfies these conditions then every finitely presented module in $\sigma[P]$ is embedded in $P^{(\mathbb{N})}$.

Proof. $(a) \Rightarrow(b)$ By assumption $P^{k} / K$ is $P$-presented and hence we have an exact diagram

$$
\begin{aligned}
& 0 \rightarrow K \rightarrow P^{k} \rightarrow P^{k} / K \rightarrow 0 \\
& \beta \vdots \quad \alpha \vdots \quad \| \\
& 0 \rightarrow L \rightarrow P^{(\Lambda)} \rightarrow P^{k} / K \rightarrow 0,
\end{aligned}
$$

where $L$ is $P$-generated. The projectivity of $P$ yields some $\alpha: P^{k} \rightarrow P^{(\Lambda)}$, and then there exists $\beta: K \rightarrow L$ extending the diagram commutatively. By the weak injectivity of $L$ we obtain some morphism $P^{k} \rightarrow L$ which shows that the lower sequence splits (by the Homotopy Lemma). So $P^{k} / K \in \operatorname{Add}(P)$.

$(b) \Rightarrow(a)$ Let $N$ be a $P$-generated module. For any finitely generated $K \subset P^{k}$ and $f: K \rightarrow N$, we have - by a pushout construction - the commutative exact diagram

$$
\begin{aligned}
& 0 \rightarrow K \rightarrow P^{k} \rightarrow P^{k} / K \rightarrow 0 \\
& 0 \rightarrow N \rightarrow Q \rightarrow P^{k} / K \rightarrow 0 \text {. }
\end{aligned}
$$

Since $P^{k} / K \in \operatorname{Add}(P), \operatorname{Ext}_{P}^{1}\left(P^{k} / K, N\right)=0$ and the lower sequence splits. This proves that $N$ is weakly $P$-injective. 
Now assume that $P$ is a direct summand of a direct sum of finitely presented modules in $\sigma[P]$.

$(a) \Rightarrow(c) \operatorname{Gen}(P)=\mathrm{w}-\operatorname{Inj}(M)$ implies that $P$ is semi-cohereditary and that any direct limit of weakly $M$-injective modules is weakly $M$-injective. Hence $P$ is locally coherent by 6.1 .

$(c) \Rightarrow(b)$ By 6.4, $\operatorname{Gen}(P)=\mathrm{w}-\operatorname{Inj}(P)$. Since $P$ is pure projective, $\operatorname{Gen}(P) \subset P^{\perp_{P}}$.

The final assertion is clear by the fact that every finitely presented module in $\sigma[P]$ is submodule of some $P^{k} / K, K$ finitely generated (see $[19,25.1]$ ).

The conditions of 6.9 are obviously satisfied by the modules considered in 6.5 - 6.8. Moreover any projective module over a von Neumann regular ring satisfies $6.9(\mathrm{c})$.

\section{Cohereditary coalgebras}

In this section $R$ denotes a commutative ring. Let $\Delta: C \rightarrow C \otimes_{R} C$ be a coalgebra over $R$ with counit $\varepsilon: C \rightarrow R$, and Comod- $C$ the category of right $C$-comodules. For basic properties of coalgebras we refer to [21].

$C$ is a subgenerator in Comod-C and Comod-C is a Grothendieck category if and only if $C$ is flat as an $R$-module.

$C^{*}=\operatorname{Hom}_{R}(C, R)$ is an $R$-algebra and every right $C$-comodule is a left $C^{*}$ module, and $C o m o d-C$ is a subcategory of $C^{*}$-Mod. If $C$ is projective as an $R$-module then $C o m o d-C$ is a full subcategory of $C^{*}$-Mod with subgenerator $C$, i.e.

$$
\text { Comod- } C=\sigma\left[C^{*} C\right] .
$$

Definition. We call a coalgebra $\mathrm{C}$ right cohereditary if every factor of $C$ by a right coideal is injective in $\operatorname{Comod}$-C. If $C_{R}$ is projective this is equivalent to the property that every factor of $C$ by a left $C^{*}$-submodule is injective as $C^{*}$-module, i.e., $C$ is cohereditary in $\sigma\left[C^{*} C\right]$.

Assume $C_{R}$ to be projective. Then the Finiteness Theorem for comodules says that every finite subset of a right $C$-comodule $M$ is contained in a subcomodule which is finitely gnerated as $R$-module. This implies that over a noetherian (artinian) ring $R, C$ is a locally noetherian (artinian) left $C^{*}$-module.

It is also known that over any self-injective $\operatorname{ring} R, C$ is injective in $\sigma\left[C^{*} C\right]$, and over a noetherian injective ring $R$ (=QF-ring), every module in $\sigma\left[C^{*} C\right]$ is a submodule of some direct sum $C^{(\Lambda)}$.

Hence 6.3 yields the following characterization of cohereditary coalgebras. 


\subsection{Right cohereditary coalgebras.}

Let $R$ be a (noetherian) QF-ring and $C$ an $R$-coalgebra with $C_{R}$ projective.

(1) The following are equivalent:

(a) $C$ is a right cohereditary coalgebra;

(b) $C$ is cohereditary in $\sigma\left[C^{*} C\right]$;

(c) every injective module is cohereditary in $\sigma\left[\mathrm{C}^{*} C\right]$;

(d) every indecomposable injective module is cohereditary in $\sigma\left[{ }_{C^{*}} C\right]$ :

If these conditions are satisfied, $C$ is tilting in $\sigma\left[{ }_{C^{*}} C\right]$.

(2) The following are equivalent:

(a) $C$ is a right cohereditary coalgebra and $C$ is finitely generated as $C^{*}$ module;

(b) $C^{*}$ is a left hereditary algebra and $\sigma\left[{ }_{C^{*}} C\right]=C^{*}$-Mod;

(c) $C$ is tilting in $C^{*}-\operatorname{Mod}$ and $\operatorname{Gen}(C)=\operatorname{Inj}(C)$.

Proof. (1) $(a) \Leftrightarrow(b)$ is clear by the correspondence between right comodules and left $C^{*}$-modules; $(b) \Leftrightarrow(c) \Leftrightarrow(d)$ follows from 6.3.

In the situation given, $C$ is tilting in $\sigma\left[{ }_{C^{*}} C\right]$ by 6.5 since $C$ is a cogenerator in $\sigma\left[{ }_{C^{*}} C\right]$ and locally of finite length.

(2) If $C$ is finitely generated as $C^{*}$-module then, by the Finiteness Theorem, $C$ is finitely generated as $R$-module which means $\sigma\left[{ }_{C^{*}} C\right]=C^{*}-M o d$, i.e., $C$ is a subgenerator in $C^{*}$-Mod. Now the assertions follow from 6.3 and 6.5.

Remarks. Cohereditary coalgebras over fields were considered in NăstăsescuTorrecillas-Zhang [16] (under the name hereditary coalgebras). Our characterization 7.1 extends most of their Theorem 4 from base fields to QF-rings. Since in 7.1, $C$ has locally finite length as $C^{*}$-module, their proof $(2) \Rightarrow(1)$ applies to show that $(a)$ is equivalent to

$$
\operatorname{inj} \cdot \operatorname{dim}(S) \leq 1 \text {, for any simple } S \in \sigma\left[{ }_{C^{*}} C\right] \text {, }
$$

where the injective dimension is considered in $\sigma\left[{ }_{C^{*}} C\right]$.

Acknowledgement. This paper was written during a stay of the author at the Department of Mathematics of the University of Lisbon. He wants to express his gratitude for the kind hospitality and the financial support he received. Moreover he is indebted to R. Colpi, W. Folz and A. Facchini for stimulating discussions on the topic. In particular W. Folz's diploma thesis [11] was very helpful for finding the way through the material. 


\section{References}

[1] I. Assem, Tilting Theory - An Introduction, Banach Center Publ., Topics in Algebra 26 (I) (1990), 127-180.

[2] R. Colpi, Some remarks on equivalences between categories of modules, Comm. Algebra 18 (6) (1990), 1935-1951.

[3] R. Colpi, Tilting modules and *-modules, Comm. Algebra 21 (4) (1993), 1095-1102.

[4] R. ColPI, Tilting in Grothendieck categories, Preprint (1997).

[5] R. Colpi And C. Menini, On the structure of *-modules, J. Algebra 158 (1993), 400-419.

[6] R. Colpi and J. Trlifaj, Tilting modules and tilting torsion theories, J. Algebra 178 (1995), 614-634.

[7] R. Colpi, G. D'Este And A. Tonolo, Quasi-tilting modules and counter equivalences, J. Algebra 191 (1997), 461-494.

[8] R. Colpi, A. Tonolo and J. Trlifaj, Partial cotilting modules and the lattices induced by them, Preprint.

[9] A. Facchini, Divisible modules over integral domains, Arkiv Math. 26 (1988), 67-85.

[10] A. FACCHInI, A tilting module over commutative integral domains, Comm. Algebra 15 (11) (1987), 2235-2250.

[11] W. Folz, Zur Theorie der *-Moduln und Kipp-Moduln, Diplomarbeit, Universität Düsseldorf (1997).

[12] L. FuCHS, On divisible modules over domains, CISM Courses and Lectures 287 (1984), 341-356.

[13] J. Golan, T. Head, Modules and the Structure of Rings, Marcel Dekker, New York (1991).

[14] D. Happel and C. M. Ringel, Tilted algebras, Trans. Amer. Math. Soc. 274 (2) (1982), 399-443

[15] C. Menini and A. Orsatti, Representable equivalences between categories of modules and applications, Rend. Sem. Mat. Univ. Padova 82 (1989), 203-231. 
[16] C. NǍstǎsescu, B. Torrecillas, Y.H. Zhang, Hereditary coalgebras, Comm. Algebra 24 (4) (1996), 1521-1528.

[17] J. TrLifaJ, On $*$-modules generating the injectives, Rend. Sem. Mat. Univ. Padova 88 (1992), 211-220.

[18] J. TRLifAJ, Every *-module is finitely generated, J. Algebra 169 (1994), 392-398.

[19] R. Wisbauer, Foundations of Module and Ring Theory, Gordon and Breach, Reading (1991).

Grundlagen der Modul- und Ringtheorie, Verlag R. Fischer, München (1988).

[20] R. Wisbauer, On module classes closed under extensions, Rings and radicals, Gardner, Liu Shaoxue, Wiegandt (ed.), Pitman RN 346, 73-97 (1996).

[21] R. Wisbauer, Introduction to Coalgebras and Comodules, Lecture Notes, Düsseldorf (1997). 